\title{
Clinical and Spectral-Domain Optical Coherence Tomography Appearance of Optic Disc Melanocytoma: A New Classification and Differentiation from Pigmented Choroidal Lesions
}

\author{
Supanut Apinyawasisuk ${ }^{\mathrm{a}}$ Tara McCannel $^{\mathrm{b}}$ Anthony C. Arnold ${ }^{\mathrm{b}}$ \\ a Department of Ophthalmology, Chulalongkorn University, Bangkok, Thailand; bUCLA Stein Eye Institute, \\ Los Angeles, CA, USA
}

\section{Key Words}

Optic disc melanocytoma $\cdot$ Spectral-domain optical

coherence tomography . Choroidal lesions

\begin{abstract}
Aims: The aim of this paper was to compare the features of both the classic, darkly pigmented and the atypical, more lightly pigmented optic disc melanocytoma with those of pigmented choroidal lesions. Methods: We analyzed the spectral-domain optical coherence tomography (SD-OCT) features of 9 eyes with optic disc melanocytoma and compared them with those of choroidal melanoma and nevus. Results and Conclusion: We identified 2 categories of SDOCT findings in optic disc melanocytoma: (a) type 1, the typical, prominent, hyperpigmented lesion with SD-OCT findings of a hyperreflective, disorganized overlying retina and a posterior hyporeflective shadow, and (b) the less common, atypical, minimally pigmented type 2 lesion overlaid by a relatively well-organized retina that lacks a posterior hyporeflective shadow. Choroidal lesions were characterized by tumor confined beneath the clearly visible hyperreflective line of the photoreceptor and retinal pigment epithelium, with minimal disorganization of the overlying retina.
\end{abstract}

(c) 2016 S. Karger AG, Basel

\section{KARGER}

(๑) 2016 S. Karger AG, Basel

E-Mail karger@karger.com

www.karger.com/oop

\section{Introduction}

Optic disc melanocytoma is a benign, typically darkbrown- to black-pigmented tumor located within or adjacent to the optic disc. The tumor is usually stable or demonstrates a very slow growth. The primary differential diagnosis includes juxtapapillary choroidal melanoma or nevus. These may be clinically distinguished in many cases by their deeper location and the presence of subretinal fluid or orange pigmentation. Additionally, ancillary testing such as ultrasonography and fluorescein angiography may aid in determination. However, differentiating a benign optic disc melanocytoma from a malignant melanoma may remain challenging in some cases, particularly when the melanocytoma is less pigmented or has subretinal fluid associated. Distinguishing the 2 entities is critical from a management standpoint.

Spectral-domain optical coherence tomography (SDOCT) is widely used for the diagnosis and anatomic characterization of retinal and choroidal lesions. The SDOCT features of choroidal melanoma and nevus have been well described [1-4]. While a number of case reports and small case series include limited descriptions of the corresponding features of the classic, darkly pigmented optic disc melanocytoma, these have not included the un- 
Table 1. Demographic and clinical data of 9 patients with optic disc melanocytoma

\begin{tabular}{llllllllllll}
\hline $\begin{array}{l}\text { Case } \\
\text { No. }\end{array}$ & $\begin{array}{l}\text { Age, } \\
\text { years/sex }\end{array}$ & Eye & VA & APD & $\begin{array}{l}\text { VF pattern/ } \\
\text { MD, dB }\end{array}$ & Size, DD & Type & ODE & OA & SRF & BRVO \\
\hline 1 & $14 / \mathrm{F}$ & OS & $20 / 30$ & Yes & EBS/-3.2 & 0.5 & 2 & No & No & No & No \\
2 & $58 / \mathrm{M}$ & OD & $20 / 20$ & No & PD/-21.42 & 1.0 & 1 & Yes & No & No & No \\
3 & $42 / \mathrm{M}$ & OD & $20 / 20$ & No & n.a. & 3.0 & 2 & No & No & No & No \\
4 & $44 / \mathrm{M}$ & OD & $20 / 20$ & No & NL/-0.67 & 1.0 & 1 & No & No & No & Yes \\
5 & $33 / \mathrm{M}$ & OD & $20 / 400$ & No & n.a. & 2.0 & 1 & No & No & No & No \\
6 & $48 / \mathrm{M}$ & OD & $20 / 30$ & No & n.a. & 1.2 & 1 & No & No & No & No \\
7 & $24 / \mathrm{F}$ & OS & $20 / 15$ & Trace & n.a. & 5.0 & 1 & No & No & No & No \\
8 & $21 / \mathrm{M}$ & OS & $20 / 20$ & No & n.a. & 1.5 & 1 & No & No & No & No \\
9 & $42 / \mathrm{F}$ & OS & $20 / 20$ & No & EBS/-2.68 & 1.0 & 1 & No & No & No & No \\
\hline
\end{tabular}

VA, visual acuity; APD, afferent pupillary defect; VF, visual field; MD, mean deviation; DD, disc diameter; ODE, optic disc edema; OA, optic atrophy; SRF, subretinal fluid; BRVO, branch retinal vein occlusion; OD, right eye; OS, left eye; EBS, enlarged blind spot; PD, peripheral depression; n.a., not available; NL, normal.

common, more lightly pigmented form of the tumor [519]. We compared the features of both the classic, darkly pigmented and the atypical, more lightly pigmented optic disc melanocytoma with those of pigmented choroidal lesions.

\section{Methods}

A computer search was performed of patients with the diagnosis of optic disc melanocytoma seen at the Jules Stein Eye Institute, University of California, Los Angeles Department of Ophthalmology, Divisions of Neuro-Ophthalmology and Ocular Oncology, from 1999 to 2015. A patient's diagnosis was made on the basis of previously described clinical, fluorescein angiographic, and ultrasonographic features. Of those identified, cases with high-quality SD-OCT were selected for detailed study. In cases of an initial diagnosis prior to the availability of SD-OCT, the study was performed at routine follow-up examinations.

Demographic and clinical data were collected, including age, sex, involved eye, best-recorded visual acuity, presence of relative afferent pupillary defect, quantitative perimetry (Humphrey field analyzer II; Carl Zeiss Meditec, Inc., Dublin, CA, USA), tumor diameter, presence of optic disc edema, atrophy, subretinal fluid, retinal vascular occlusion, and SD-OCT images. SD-OCT of the optic nerve head was performed using Spectralis OCT (Heidelberg Engineering, Heidelberg, Germany). Horizontal scans were performed using 19- to 25-line raster acquisition patterns. The interval between each slice in the raster acquisition patterns was 240 microns. The scanned area was adjusted to cover the tumor and the adjacent retinal and optic nerve head tissue. Only a single-line scan through the tumor was available in some patients.

One case each of choroidal melanoma and choroidal nevus with typical clinical, fluorescein angiographic, ultrasonographic, and SD-OCT imaging features were selected for direct comparison by identical OCT imaging techniques.

Clinical and SD-OCT Appearance of

Optic Disc Melanocytoma

\section{Results}

Demographic and clinical data are summarized in Table 1 .

Nine eyes of 9 patients with optic disc melanocytoma were included. Age ranged from 14 to 58 years, and 6 patients were male. Best-corrected visual acuity ranged from $20 / 15$ to $20 / 400$ in the involved eye. A relative afferent pupillary defect was present in 2 patients. Quantitative perimetry was performed in 4 patients; the mean deviation ranged from -0.67 to $-21.42 \mathrm{~dB}$. Tumor diameter ranged from 0.5 to 5.0 disc diameters. Branch retinal vein occlusion was found in 1 patient. One case showed clinically evident optic disc edema; none showed clinical evidence of subretinal fluid.

SD-OCT images of all optic disc melanocytomas demonstrated a highly elevated mass overlaid with an irregular hyporeflective and hyperreflective layer of disorganized retinal tissue (Fig. 1b) without identifiable retinal layers. At the junction between the tumor margin and the normal retina, imaging revealed a gradually elevated portion of the retina, including the retinal nerve fiber layer through the outer plexiform layer. The external limiting membrane and ellipsoid zone were attached to the underlying retinal pigment epithelium without elevation from mass effect (Fig. 1b). There were scattered hyperreflective dots (Fig. 1b, d) in the overlying retina. Minimal subretinal fluid, not appreciable in the clinical examination, was identified adjacent to the tumor in 1 patient.

The SD-OCT findings for each patient are summarized in Table 2. 

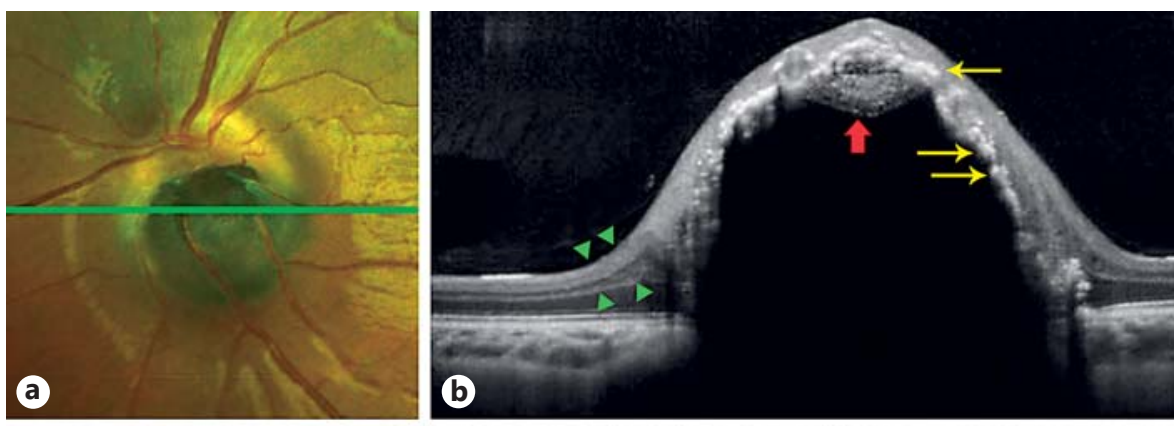

Fig. 1. Fundus photograph $(\mathbf{a}, \mathbf{c})$ and SDOCT image $(\mathbf{b}, \mathbf{d})$ of a heavily pigmented optic disc melanocytoma. The SD-OCT image shows a highly elevated mass with a thin, disorganized overlying retina and underneath a dense hyporeflective shadow. Yellow arrows indicate hyperreflective dots scattered all over the retina with accumulation at the tumor-retina interface. Tubular structures demonstrate major retinal vessels (red vertical arrow). Inner retinal layers (from the retinal nerve fiber layer to the outer plexiform layer) are gradually elevated by mass effect (double green arrowheads). A fundus photograph $(\mathbf{e}, \mathbf{g})$ and SD-OCT image $(\mathbf{f}, \mathbf{h})$ of a lightly pigmented optic disc melanocytoma. A thicker and more organized overlying retina is shown. The hyporeflective shadow and tumor interface are not well visualized. Fewer hyperreflective dots (yellow arrows) are scattered within the retina without accumulation. Inner retinal layers (from the retinal nerve fiber layer to the outer plexiform layer) are gradually elevated by mass effect as seen in heavily pigmented lesions (double green arrowheads). SD-OCT, spectraldomain optical coherence tomography; ODM, optic disc melanocytoma; RNFL, retinal nerve fiber layer; OPL, outer plexiform layer.
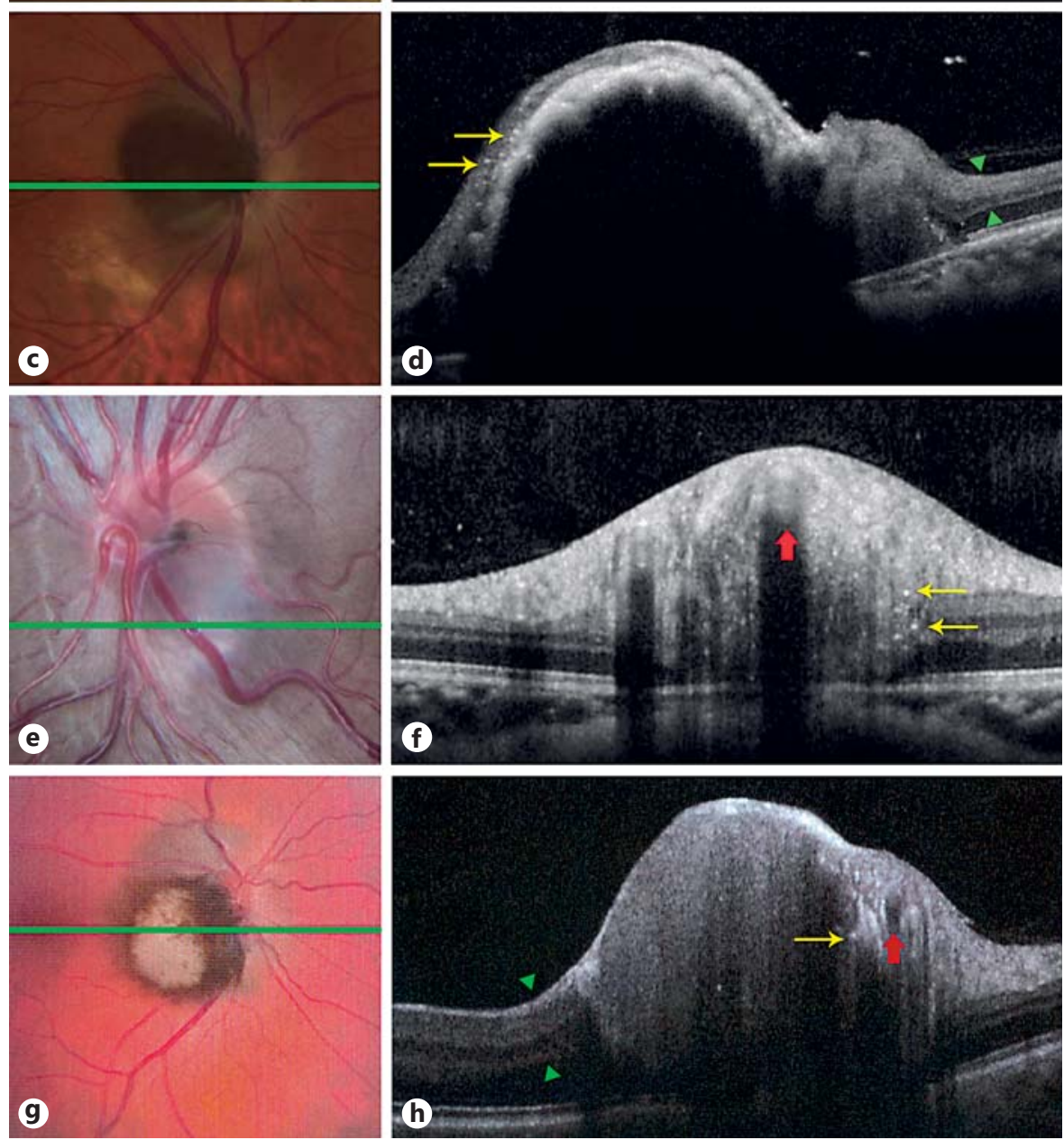

Two distinct sets of SD-OCT features of optic disc melanocytoma were identified. Type 1 , the most heavily pigmented lesion type, demonstrated a deep hyporeflective shadow caused by blockage from the tumor pigment, with thin overlying retinal tissue. The interface between the tumor and the retina was sharp and well defined. The distribution of intraretinal hyperreflective dots was dense and was localized at the tumor-retina interface (Fig. 1b). Seven patients were classified as Type 1 . Type 2, the less pigmented type of lesion, demonstrated a hyperreflective shadow extending deeply into the tumor mass, containing scattered hyperreflective dots. The interface between the tumor and the retina could not be clearly identified, and the SD-OCT signal was homogenous through the entire thickness of the lesion (Fig. 1d). There was no accumulation of hyperreflective dots as noted in Type 1 optic disc melanocytoma. Two patients were classified as Type 2. Clinically, Type 2 optic disc melanocytoma was located deeper and was relatively less pigmented than the Type 1 lesion. 
Fig. 2. Fundus photograph (a) and SDOCT image (b) of a choroidal melanoma. The SD-OCT image shows choroidal elevation with optical shadowing. Intraretinal edema, thickening of the photoreceptor layer, and rare, hyperreflective dots (yellow arrows) are seen in the organized overlying retina. In the choroidal nevus (c), SD-OCT (d) demonstrates an elevated choroidal lesion with mild optical shadowing and moderate retinal pigment epithelial thickening. The overlying retinal layers are elevated by the effect of drusenoid pigment epithelial detachment. SD-OCT, spectraldomain optical coherence tomography; RPE, retinal pigment epithelium; PED, pigmented epithelial detachment.
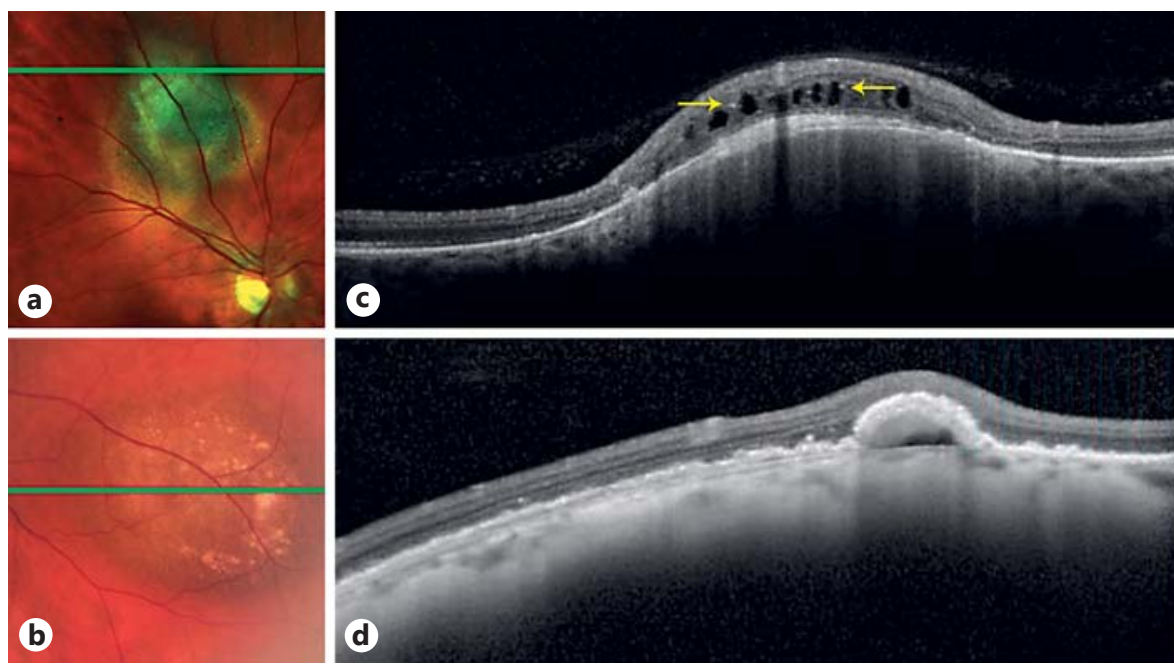

Table 2. SD-OCT findings of 9 patients with optic disc melanocytoma

\begin{tabular}{|c|c|c|c|c|c|c|c|}
\hline $\begin{array}{l}\text { Case } \\
\text { No. }\end{array}$ & Type & Overlying retina & $\begin{array}{l}\text { Hyporeflective } \\
\text { shadow }\end{array}$ & $\begin{array}{l}\text { Separation of } \\
\text { retinal layers }\end{array}$ & $\begin{array}{l}\text { Tumor-retina } \\
\text { interface }\end{array}$ & Hyperreflective dots & $\begin{array}{l}\text { Tumor } \\
\text { location }\end{array}$ \\
\hline 1 & 2 & Organized, hyperreflective & Absent & Not seen & Ill defined & Less prominent, scattered & Inner retina \\
\hline 2 & 1 & Disorganized, hyperreflective & Present & RNFL to IPL & Well defined & Prominent, accumulated & Outer retina \\
\hline 3 & 2 & Organized, hyperreflective & Absent & RNFL to IPL & Ill defined & Less prominent, scattered & Unidentifiable \\
\hline 4 & 1 & Disorganized, hyperreflective & Present & RNFL to IPL & Well defined & Prominent, accumulated & Outer retina \\
\hline 5 & 1 & Disorganized, hyperreflective & Present & RNFL to IPL & Well defined & Prominent & Outer retina \\
\hline 6 & 1 & Disorganized, very thin hyperreflective & Present & Not seen & Well defined & Not seen & Outer retina \\
\hline 7 & 1 & Disorganized, hyperreflective & Present & RNFL to IPL & Well defined & Prominent, accumulated & Outer retina \\
\hline 8 & 1 & Disorganized, hyperreflective & Present & RNFL to IPL & Well defined & Prominent, accumulated & Outer retina \\
\hline 9 & 1 & Disorganized, hyperreflective & Present & RNFL to IPL & Well defined & Prominent, accumulated & Outer retina \\
\hline
\end{tabular}

SD-OCT, spectral-domain optical coherence tomography; RNFL, retinal nerve fiber layer; IPL, inner plexiform layer.

In choroidal melanoma, SD-OCT showed choroidal elevation with optical shadowing. The overlying retina remained organized without invasion of the tumor. Intraretinal edema and a thickened (shaggy) photoreceptor layer were observed over the elevated choroidal mass. Rare hyperreflective dots were found (Fig. 2a). In the choroidal nevus, the SD-OCT scan showed an elevated choroidal lesion with mild optical shadowing and a moderately thickened, irregular overlying retinal pigment epithelium. There were drusenoid pigment epithelium detachments over the nevus. Neither subretinal fluid nor intraretinal edema was observed (Fig. 2b).

\section{Discussion}

The OCT findings in optic disc melanocytoma have been described in a limited number of cases, only some of which were studied using SD-OCT. These are summarized in Table 3 [5-19]. Our cases corroborate a constel- lation of findings that have previously been reported and that are typical of most heavily pigmented optic disc melanocytomas: a very prominent and dense optical shadowing by the heavily pigmented tumor; a sharply demarcated interface between the tumor and a thin, hyperreflective layer of the disorganized overlying retina, and hyperreflective dots accumulated at the tumor-retina interface. Additionally, we noted a characteristic partial thickness elevation of the retinal tissue (from the outer plexiform layer to the retinal nerve fiber layer) at the tumor base, becoming a disorganized retina along the tumor surface. In patients with less pigmented lesions, we identified a second set of OCT features, which we describe below.

We propose 2 categories of optic disc melanocytoma based on SD-OCT imaging features. Type 1 optic disc melanocytoma is characterized by a deep hyporeflective shadow below the sharply demarcated interface between the tumor and the thin, disorganized overlying retina. Type 2 optic disc melanocytoma is characterized by a 
Table 3. Previous studies of OCT in ODM

\begin{tabular}{|c|c|c|c|c|c|}
\hline First author [ref.] & Year & Study design & $\mathrm{N}$ & OCT & OCT findings \\
\hline Chaudhary [5] & 2006 & Case report & 1 & TD-OCT & $\begin{array}{l}\text { Single hyperreflective band continued with the adjacent retina } \\
\text { and optical shadow behind the band }\end{array}$ \\
\hline Shields et al. [6] & 2006 & Retrospective review & 1 & TD-OCT & $\begin{array}{l}\text { Hyperreflective anterior surface with abrupt, dense } \\
\text { posterior shadowing }\end{array}$ \\
\hline Shields [7] & 2008 & Retrospective review & 15 & TD-OCT & $\begin{array}{l}\text { Gradually sloped nodular elevation with a hyperreflective } \\
\text { anterior surface with abrupt, dense posterior shadowing (14 } \\
\text { cases) and without posterior shadowing ( } 1 \text { case) }\end{array}$ \\
\hline Shah $[8]$ & 2009 & Case report & 1 & $\begin{array}{l}\text { TD- and } \\
\text { SD-OCT }\end{array}$ & $\begin{array}{l}\text { Hyperreflective signal with optical shadowing (TD-OCT), } \\
\text { high reflectivity, and focal thickening of RPE (SD-OCT) }\end{array}$ \\
\hline Finger [9] & 2010 & Retrospective review & 20 & TD-OCT & $\begin{array}{l}\text { RNFL hyperreflectivity ( } 12 \text { eyes), choroidal } \\
\text { hyperreflectivity, or RPE upward displacement (14 eyes), } \\
\text { correlation with histopathologic report in } 1 \text { case }\end{array}$ \\
\hline Saxena [10] & 2010 & Case report & 1 & SD-OCT & $\begin{array}{l}\text { Elevated hyperreflective line corresponding to the tumor } \\
\text { surface with dense optical shadowing }\end{array}$ \\
\hline Zalilawati [11] & 2011 & Case report & 1 & SD-OCT & Hyperreflective band overlying limited optical shadowing \\
\hline Mazzuca [12] & 2012 & Retrospective review & 2 & SD-OCT & $\begin{array}{l}\text { Elevated hyperreflective line corresponding to the tumor } \\
\text { surface with dense optical shadowing }\end{array}$ \\
\hline Okubo [13] & 2013 & Retrospective review & 5 & SD-OCT & $\begin{array}{l}\text { Irregularly wavy anterior surface, variously sized, } \\
\text { hyperreflective dots distributed in the retina and } \\
\text { accumulated around the vessels; dense posterior shadowing }\end{array}$ \\
\hline Guerra [14] & 2014 & Retrospective review & 2 & SD-OCT & $\begin{array}{l}\text { Elevated hyperreflective line corresponding to the tumor } \\
\text { surface with dense optical shadowing }\end{array}$ \\
\hline Batmanabane [15] & 2015 & Case report & 1 & SD-OCT & $\begin{array}{l}\text { Elevated hyperreflective band with optical shadowing; } \\
\text { adjacent subretinal fluid }\end{array}$ \\
\hline Eldaly [16] & 2015 & Case report & 1 & SD-OCT & $\begin{array}{l}\text { Dome-shaped, elevated lesion with a highly reflective layer of } \\
\text { the tumor mass, continuous with RNFL with optical } \\
\text { shadowing }\end{array}$ \\
\hline Lee $[17]$ & 2015 & Retrospective review & 1 & SD-OCT & $\begin{array}{l}\text { Moderately thinned hyperreflective band with optical } \\
\text { shadowing }\end{array}$ \\
\hline Salvanos [18] & 2015 & Retrospective review & 1 & SD-OCT & $\begin{array}{l}\text { Moderately thinned hyperreflective band with optical } \\
\text { shadowing }\end{array}$ \\
\hline Zhang [19] & 2016 & Retrospective review & 8 & SD-OCT & $\begin{array}{l}\text { Band of disorganized hyperreflective retina overlying } \\
\text { optical shadowing ( } 8 \text { cases); choroidal displacement ( } 8 \\
\text { cases) }\end{array}$ \\
\hline
\end{tabular}

OCT, optical coherence tomography; ODM, optic disc melanocytoma; N, number of patients; TD, time domain; SD, spectral domain; RPE, retinal pigment epithelium; RNFL, retinal nerve fiber layer.

blending, hyperreflective shadow extending to the outer retinal layers without sharp demarcation of the tumorretina interface.

Most previous reports demonstrated tumors with the typical dense, hyporeflective shadow of more heavily pigmented lesions (classified as Type 1 in our study). How- ever, in a series of 15 optic disc melanocytoma patients studied with early-generation SD-OCT reported by Shields et al. [7], 1 patient with a small, flat melanocytoma within the disc had no "optical shadowing of posterior layer". Though the OCT image of that patient was not shown in their study, this patient could have had shared 
features consistent with our Type 2 optic disc melanocytoma classification, if higher-quality SD-OCT had been performed. Similarly, a case reported by Zalilawati et al. [11] was more lightly pigmented and showed less optical shadowing. We propose that the Type 2 appearance may be the result of fewer signal-blocking pigmented cells within the tumor, resulting in less hyporeflective shadow and more visible intralesional structures. The hyperreflective appearance of the overlying retina may be explained by partial tumor infiltration of the less disorganized overlying retina as opposed to being displaced by the tumor as in Type 1, more heavily pigmented lesions.

SD-OCT of all optic disc melanocytoma showed scattered hyperreflective dots distributed throughout the overlying retina. Some patients demonstrated accumulation at the tumor-retina interface or major retinal vessels. Hyperreflective dots have been reported in previous SDOCT studies of age-related macular degeneration [20], diabetic macular edema, and retinal vein occlusion, as well as optic disc melanocytoma. There has been speculation that they may represent retinal microglial cells activated by the inflammatory process in age-related macular degeneration [20], lipoprotein, or lipid-laden macrophages in diabetic macular edema and retinal vein occlusion, or melanophages, in optic disc melanocytoma [13], consistent with histopathology of optic disc melanocytoma [9]. We postulate that these dots may represent lipoprotein deposits or clumps of macrophages engulfing them due to the disruption of the blood-retinal barrier in the retina disorganized by tumor damage. Their reduced numbers in Type 2 pigmented lesions would be consistent with less damage and disorganization of the overlying retina.

Differentiating optic disc melanocytoma from juxtapapillary choroidal melanoma or nevus is critical. The SD-OCT findings of melanoma and nevus we observed were consistent with previous large series [5-19]. The heavily pigmented Type 1 optic disc melanocytoma is usually distinguishable from choroidal melanoma and choroidal nevus by funduscopic findings, but SD-OCT may help confirm the diagnosis and identify associated features, such as subretinal fluid, that could affect the clinical course. The overlying retina in choroidal melanoma and nevus is usually organized and uninvolved, whereas in optic disc melanocytoma, the retina may be infiltrated by the tumor. A common characteristic in optic disc melanocytoma is a partial thickness elevation of the retinal tissue (from the outer plexiform layer to the retinal nerve fiber layer) at the tumor base, becoming a disorganized retina along the tumor surface. Interesting- ly, external limiting membrane and the outer layers remain flat and attached to the retinal pigment epithelium. To our knowledge, this feature has not been addressed in previous optic disc melanocytoma reports and is not found in choroidal melanoma or nevus (Fig. 2d). The density of posterior optical shadowing in choroidal melanoma and nevus is less prominent than in optic disc melanocytoma. While the majority of cases in both conditions demonstrate partial optical shadowing (73\% in melanoma, 59\% in nevus) [2], in optic disc melanocytoma, optical shadowing of heavily pigmented Type 1 lesions in present and previous studies is extremely dense.

More lightly pigmented Type 2 optic disc melanocytoma is more difficult to differentiate from melanoma and nevus, since the degree of hyporeflective shadowing is smaller. However, key to the distinction is that in choroidal lesions, the tumor is confined beneath the clearly visible hyperreflective line of photoreceptor and retinal pigment epithelium, whereas in Type 2 optic disc melanocytoma, the tumor is located above the photoreceptor and retinal pigment epithelial line and partly or completely blocks the signal (Fig. 2). The disorganized overlying retina and infiltration of the tumor mass into retinal layers of Type 1 optic disc melanocytoma causing separation also aid in identifying optic disc melanocytoma.

\section{Summary}

SD-OCT is a noninvasive imaging technique that can be utilized as an ancillary test in optic disc melanocytoma for 3 main purposes: (a) confirming the diagnosis, (b) distinguishing atypical lesions from other juxtapapillary tumors, and (c) detecting subclinical features. Our study identified 2 categories of SD-OCT findings in optic disc melanocytoma: (a) Type 1, the typical, prominent, hyperpigmented lesion with SD-OCT findings of a hyperreflective, disorganized overlying retina and a posterior hyporeflective shadow, and (b) the less common, atypical, minimally pigmented Type 2 lesion overlaid by a relatively well-organized retina that lacks a posterior hyporeflective shadow.

\section{Statement of Ethics}

The study was approved by the UCLA Office for Protection of Research Subjects Institutional Review Board (IRB\#15-001339, approval date 9/2/15) for a retrospective review of patient data. The requirement for informed consent was waived. 


\section{Disclosure Statement}

The authors report that they have no conflicts of interest to disclose.

\section{References}

1 Chen N, Huang TL, Tsai RK: Spectral domain optical coherence tomography findings of juxtapapillary choroidal nevus. Neuro-Ophthalmology 2012;36:20-22.

2 Shields CL, Kaliki S, Rojanaporn D, Ferenczy SR, Shields JA: Enhanced depth imaging optical coherence tomography of small choroidal melanoma: comparison with choroidal nevus. Arch Ophthalmol 2012;130:850-856.

3 Say EA, Shah SU, Ferenczy S, Shields CL: Optical coherence tomography of retinal and choroidal tumors. J Ophthalmol 2012;2012: 385058.

4 Vishnevskia-Dai V, Zur D, Yaacobi S, Moroz I, Newman H, Neudorfer M: Optical coherence tomography: an adjunctive tool for differentiating between choroidal melanoma and metastasis. J Ophthalmol 2016;2016: 9803547.

5 Chaudhary R, Arora R, Mehta DK, Singh M: Optical coherence tomography study of optic disc melanocytoma. Ophthalmic Surg Lasers Imaging 2006;37:58-61.

6 Shields JA, Demirci H, Mashayekhai A, Eagle RC, Shields CL: Melanocytoma of the optic disc: a review. Surv Ophthalmol 2006;51:93104.

7 Shields CL, Perez B, Benavides R, Materin MA, Shields JA: Optical coherence tomography of optic disk melanocytoma in 15 cases. Retina 2008;28:441-446.
8 Shah VA, Vincent RD, Desai K, Gallimore G, Rupani M: Documentation of optic disc melanocytoma by spectral and time domain optical coherence tomography. Can J Ophthalmol 2009;44:603-604.

9 Finger PT, Natesh S, Milman T: Optical coherence tomography: pathology correlation of optic disc melanocytoma. Ophthalmology 2010;117:114-119.

10 Saxena S, Sharma B, Bhasker SK: Three-dimensional spectral-domain optic coherence tomography of melanocytoma of the optic nerve head. J Ocul Biol Disc Inform 2010;3: 112-116.

11 Zalilawati M, Kah TA, Yong KC, Abdul Halim WHW, Yong TK: Melanocytoma of the optic nerve head-a diagnostic dilemma. Clin Pract 2011;1:e60:123-125.

12 Mazzuca DE, Shields CL, Sinha N, Bianciotto CE, Fox G, Shields JA: Progressive retinal invasion and vitreous seeding from optic disc melanocytoma. Clin Exp Ophthalmol 2012; 40:e123-e125.

13 Okubo A, Unoki K, Yoshikawa H, Ishibashi T, Sameshima M, Sakamoto T: Hyperreflective dots surrounding the central retinal artery and vein in optic disc melanocytoma revealed by spectral domain optical coherence tomography. Jpn J Ophthalmol 2013;57:108112.

14 Guerra RL, Marback EF, Silva IS, Maia Junior Ode O, Marback RL: Autofluorescence and spectral-domain optical coherence tomography of optic disk melanocytoma. Arq Bras Oftalmol 2014;77:400-402.
15 Batmanabane V, Soman M, Nair U: Management of optic disc melanocytoma associated with choroidal neovascularisation and macular detachment. J Ophthalmic Clin Res 2015, 2:017.

16 Eldaly H, Eldaly Z: Melanocytoma of the optic nerve head, thirty-month follow-up. Semin Ophthamol 2015;21:1-6.

17 Lee E, Sanjay S: Optic disc melanocytoma. Report of 5 patients from Singapore with review of literature. Asia Pac J Ophthalmol (Phila) 2015:4:273-278.

18 Salvanos P, Utheim TP, Moe MC, Eide N, Bragadomicronttir R: Autofluorescence imaging in the differential diagnosis of optic disc melanocytoma. Acta Ophthalmol 2015;93: 476-480

19 Zhang P, Hiu Y-N, Xu W-Q, Zhang Z-F, Wang H-Y, Sun D-J, Wang Y-S: Infrared autofluorescence, short-wave autofluorescence, and spectral-domain optic coherence tomography of optic disc melanocytomas. Int J Ophthalmol 2016;9:713-716

20 Coscas G, De Benedetto U, Coscas F, et al: Hyperreflective dots: a new spectral-domain optical coherence tomography entity for followup and prognosis in exudative age-related macular degeneration. Ophthalmologica 2013;229:32-37. 